\title{
Genomic evolution and complexity of the Anaphase-promoting Complex (APC) in land plants
}

\author{
Marcelo de F Lima', Núbia B Eloy ${ }^{1}$, Camila Pegoraro², Rauan Sagit ${ }^{3}$, Cristian Rojas' ${ }^{1}$, Thiago Bretz ${ }^{1}$, Lívia Vargas',
} Arne Elofsson ${ }^{3}$, Antonio Costa de Oliveira ${ }^{2}$, Adriana S Hemerly ${ }^{1}$, Paulo CG Ferreira ${ }^{1 *}$

\begin{abstract}
Background: The orderly progression through mitosis is regulated by the Anaphase-Promoting Complex (APC), a large multiprotein $E_{3}$ ubiquitin ligase that targets key cell-cycle regulators for destruction by the $26 \mathrm{~S}$ proteasome. The APC is composed of at least 11 subunits and associates with additional regulatory activators during mitosis and interphase cycles. Despite extensive research on APC and activator functions in the cell cycle, only a few components have been functionally characterized in plants.

Results: Here, we describe an in-depth search for APC subunits and activator genes in the Arabidopsis, rice and poplar genomes. Also, searches in other genomes that are not completely sequenced were performed. Phylogenetic analyses indicate that some APC subunits and activator genes have experienced gene duplication events in plants, in contrast to animals. Expression patterns of paralog subunits and activators in rice could indicate that this duplication, rather than complete redundancy, could reflect initial specialization steps. The absence of subunit APC7 from the genome of some green algae species and as well as from early metazoan lineages, could mean that APC7 is not required for APC function in unicellular organisms and it may be a result of duplication of another tetratricopeptide (TPR) subunit. Analyses of TPR evolution suggest that duplications of subunits started from the central domains.

Conclusions: The increased complexity of the APC gene structure, tied to the diversification of expression paths, suggests that land plants developed sophisticated mechanisms of APC regulation to cope with the sedentary life style and its associated environmental exposures.
\end{abstract}

\section{Background}

Cell proliferation is controlled by an universally conserved molecular machinery in which the key players are cyclin-dependent kinases (CDK) and cyclins (reviewed in [1]). Eukaryotes have therefore evolved elaborate mechanisms for CDK regulation. An irreversible mechanism of CDK down-regulation is destruction of cyclin subunits $[2,3]$. At the $\mathrm{G}_{1}$ - to $\mathrm{S}$-phase and metaphase to anaphase transitions, CDKs are irreversibly inactivated by ubiquitin-mediated proteolysis of cognate cyclins [4]. Degradation of protein substrates through the ubiquitin-proteasome pathway involves the activity of different E3 ligases, among them the anaphase-

\footnotetext{
* Correspondence: paulof@bioqmed.ufrj.br

Full list of author information is available at the end of the article
}

promoting complex (APC). The APC was first identified based on its role in facilitating the multiubiquitination and targeting of A- and B-type cyclins for proteasomemediated destruction during mitosis [5-8]. APC is a multiprotein complex conserved from plants to man and contains at least 11 core subunits [9]. The APC is regulated in part by two associated proteins, $\mathrm{CDC} 20$ and CCS52/CDH1, that can both activate the APC with proper timing and provide substrate specificity $[10,11]$. The APC is activated at metaphase/anaphase transition by the $\mathrm{CDC} 20$ protein and later in telophase and $\mathrm{G}_{1}$ by the $\mathrm{CDH} 1$ protein. Substrates that have a destruction box (D-box), KEN-box or A-box motifs are recognized and ubiquitinated by the APC [12-14].

The APC has important functions in mitosis, meiosis, $\mathrm{G}_{1}$-S-phase and in post-mitotic differentiated cells 
$[15,16]$. The mitosis-specific activator CDC20 is itself an APC substrate. Other targets of APC/C degradation are: Cyclins A and B; protein kinases Plk1, CDC5, Aurora A and $B$; regulators of DNA replication Geminin, CDC6; and the anaphase inhibitor Securin (reviewed in [17]). The proteolytic events triggered by the APC are required to release sister chromatides cohesion during anaphase, to control the exit from mitosis and to prevent premature entry into S-phase $[6,18,19]$.

Although the role of the APC in controlling cell-cycle progression has been extensively investigated, has recent work shown the unexpected presence of APC subunits in differentiated tissues, in particular in the nervous system of vertebrates (reviewed in [20]). In plants, the expression of several APC subunits has been detected in differentiated tissues of Arabidopsis [21,22]. In addition, while both $C D C 27 \mathrm{a}$ and $\mathrm{CDC} 27 \mathrm{~b}$ genes are essential during gametogenesis, the $\mathrm{CDC} 27 \mathrm{~b}$ subunit has been implicated also in post-embryogenic differentiation at meristem levels [23]. Besides, it has been shown that reduced levels of APC6 and APC10 subunits in Arabidopsis lead to plants with several defects in vascular development [24]. These results indicate that, while the overall structure of the APC is conserved among eukaryotes, this E3 ligase may have assumed specialized functions in the diverse kingdoms. Gene duplication and retention in plants has been extensive and gene families are generally larger in plants than in animals [25]. Nevertheless, almost all studies on the APC function in plants have been carried out in the model plant Arabidopsis thaliana. Comparative genomic analyses can provide valuable insights into the organization of cell cycle machinery and the evolution of these protein complexes.

In this article, we describe thorough searches for the predicted sequences of the APC subunits and the activators CDC20/CCS52 in plant sequence databases. Our results indicate that land plants and green algae orthologs have an ancient evolutionary origin. We present phylogenetic analysis of TPR subunits and activators, and their orthologs from other plants. An evolutionary analysis of Arabidopsis TPR domain suggests that tandem regions could have been created from duplication of internal sequence domains. Overall, our data support the proposal that APC subunits and activators have been conserved in the course of evolution. However, while other eukaryotes like fungi and metazoans have only one copy of each APC subunit and its regulators, gene duplication of different subunits have occurred in Arabidopsis, rice and poplar, and in other plant genomes as well. An attractive proposal is that gene duplication of APC subunits may result in the formation of APC subcomplexes that evolved to assume restricted specialized roles during plant development. Indeed, in Arabidopsis, duplication of the CDC27 subunit led to substantial sequence divergence and specialization [23]. Here we show that the two copies of rice CDC23 and APC11 subunits and the activators genes are differentially expressed in plant tissues or when grown under either dark or light-mediated developmental programs, suggesting the duplicated genes could be assuming new functions in the plant.

\section{Results and Discussion}

\section{Identification of APC subunits and activator genes}

In order to carry out the cross-species comparison of the APC and activators, we have searched for homologous sequences in the Genomic Research (TIGR) Rice genome database and DOE Join Genome Institute (JGI) Poplar genome database. The sequences of the Arabidopsis APC subunits have been published [21,26,27]. Even after exhaustive data mining, some rice and poplar sequences were only found in the EST database Gene Index DFCI. Subsequently, the SMART motif identification tool was used to identify predicted domains in all candidate proteins [28]. Using this strategy, we report the APC/activators in the dicotyledonous poplar and the monocotyledon rice plants (Table 1 and Additional file 1). All APC subunits and activators were found, and they contained the predicted conserved domains, providing one of many examples for the evolutionary conservation of the eukaryotic cell-cycle machinery [29,30].

Two CDC27 homologs have been identified in Arabidopsis $A t C D C 27 a$ and $A t C D C 27 b$ [31]. In poplar, two homologs of CDC27 were also identified: PtCDC27_1 and PtCDC27_2. However, a careful inspection of the PtCDC27_2 deposited sequence revealed a stop codon TAG at nucleotide position 1471-1473. This fragment was amplified and sequenced and the presence of the stop codon was discarded. The expression of both genes in leaves was confirmed by quantitative PCR (see Additional file 2). We also found two homologs of CDC27 in the bryophyte Physcomitrella patens genome [32]. However, only one copy of CDC27 was found in the rice genome as well as other monocot plants. On the other hand, the rice genome has two OsCDC23 and OsAPC11 subunits [33]. We examined other complete genomes and found two CDC23 ( $V v C D C 23 \_1$ and $\left.V v C D C 23 \_2\right)$ and APC11 ( $V v A P C 11 \_1$ and $\left.V v A P C 11 \_2\right)$ genes in grapevine [34]. Two homologs of PtAPC13 were found in the poplar and grapevine genomes, a feature that has not been reported in any other eukaryotic species so far.

The structures of many predicted genes were considerably misannotated. Comparing EST databases from plant species and using bioinformatics tools, it was possible to identify the correct gene sequences. The genomic sequence of OsAPC1 was not found in the TIGR database, but a partial CDS was identified in the Gene Index database (see Additional file 3). Most likely, 
Table 1 APC subunits and activators in Arabidopsis, rice and poplar genomes

\begin{tabular}{|c|c|c|c|c|}
\hline Gene Description & Arabidopsis thaliana & Oryza sativa & Populus trichocarpa & Protein Motifs \\
\hline APC Subunits & & Access number & & \\
\hline APC1 & At5g05560 & TC286185 & Pt765590 & Rpn1/2 repeats \\
\hline APC2 & At2g04660 & LOC_Os04g40830 & Pt832637 & Cullin domain \\
\hline $\mathrm{APC} 3 / \mathrm{CDC} 27 \mathrm{a}$ & At3g16320 & & & TPR repeats \\
\hline \multirow[t]{2}{*}{ APC3/CDC27b } & At2g20000 & LOC_Os06g41750 & Pt835890 & TPR repeats \\
\hline & & & Pt278795 & TPR repeats \\
\hline APC4 & At4g21530 & LOC_Os02g54490 & Pt817758 & WD-40 repeats \\
\hline APC5 & At1g06590 & LOC_Os12g43120 & Pt592813 & TPR repeats \\
\hline APC6/CDC16 & At1g78770 & LOC_Os03g13370 & Pt585761 & TPR repeats \\
\hline APC7 & At2g39090 & LOC_Os05g05720 & Pt828004 & TPR repeats \\
\hline \multirow[t]{2}{*}{ APC8/CDC23 } & At3g48150 & LOC_Os02g43920 & Pt834319 & TPR repeats \\
\hline & & LOC_Os06g46540 & & TPR repeats \\
\hline APC10 & At2g18290 & LOC_Os05g50360 & Pt796785 & Doc domain \\
\hline \multirow[t]{2}{*}{ APC11 } & At3g05870 & LOC_Os03g19059 & Pt292476 & RING-H2 domain \\
\hline & & LOC_Os07g22840 & & RING-H2 domain \\
\hline CDC26 & TC308166 & TC356501 ${ }^{a}$ & TC118342 & - \\
\hline \multirow[t]{2}{*}{$\mathrm{APC} 13$} & At1g73177 & TC311476 ${ }^{\mathrm{a}}$ & Pt647861 & \\
\hline & & & Pt660762 & - \\
\hline \multicolumn{5}{|l|}{ Activators } \\
\hline CDC20_1 & At4g33260 & LOC_Os09g06680 & Pt571123 & WD-40 repeats \\
\hline CDC20_2 & At4g33270 & LOC_Os04g51110 & Pt272847 & WD-40 repeats \\
\hline CDC20_3 & At5g26900 & LOC_Os02g47180 & Pt738273 & WD-40 repeats \\
\hline CDC20_4 & At5g27080 & & Pt256238 & WD-40 repeats \\
\hline CDC20_5 & At5g27570 & & Pt257786 & WD-40 repeats \\
\hline CDC20_6 & At5g27945 & & & WD-40 repeats \\
\hline CCS52A1 & At4g22910 & LOC_Os03g03150 & Pt415429 & WD-40 repeats \\
\hline CCS52A1_2 & & & Pt180625 & WD-40 repeats \\
\hline CCS52A2 & At4g11920 & & & WD-40 repeats \\
\hline CCS52B & At5g13840 & LOC_Os01g74146 & Pt820353 & WD-40 repeats \\
\hline CCS52B_2 & & & Pt833809 & WD-40 repeats \\
\hline
\end{tabular}

${ }^{a}$ Gene index project access number http://compbio.dfci.harvard.edu/tgi/

the largest subunit of the APC had come apart by misannotation. Diverse exon-intron structures needed correction in the genomics sequences. OsAPC4 may contain an intron in the genomic ORF and OsCDC23_2 may have a fragment of an exon involved in the formation of TPR domain erroneously annotated as an intron (see Additional file 4). The 3'region of predicted PtAPC4 and the 5'-3'regions of PtAPC5 were incomplete. However, we found fragments from 3'regions of both genes and fragments from the 5 'region of PtAPC5 in the EST database (see Additional file 4). Interestingly, OsAPC5, OsCDC16 and OsAPC11_2 have a divergent 5'region compared to Arabidopsis and poplar genes (see Additional files 4 and 5). The first methionine of OsCDC16 and OsAPC11_2 are upstream from a consensus start codon in CDC16 and APC11 genes, but only for
CDC16 there is EST support. The opposite occurs with OsAPC5, where the first methionine is downstream from the consensus start codon. The APC genes AtCDC26, OsCDC26, PtCDC26 and OsAPC13 were identified in EST databases.

Arabidopsis contains three CCS52 genes, AtCCS52A1, AtCCS52A2 and AtCCS52B; and five CDC20 genes [27]. In addition, we found another $C D C 20$ homolog in the Arabidopsis genome. This AtCDC20_6 (At5g27945) showed major differences from conserved structures of other CDC20; the CDS does not have the C-box sequence element (consensus DR(F/Y)IPxR) that was first identified in the $\mathrm{N}$-terminal region of $\mathrm{CDC} 20$, although it is conserved in all known APC/C co-activators (this was confirmed by re-sequencing - see Additional file 6), and therefore AtCDC20_6 could be a 
pseudogene. The rice genome has three predicted CDC20 genes compared to five in Poplar and Arabidopsis. Rice has two CCS52 genes compared to four and three genes in Poplar and Arabidopsis, respectively (Table 1). Exon-intron organization of activators was analyzed and we found a number of annotation errors. AtCDC20_5, PtCDC20_5, PtCCS52A1 and PtCCS52A1_2 have errors in the 5 ' region. We found that only PtCDC20_5 is without a C-box. OsCDC20_1, AtCDC20_4, PtCDC20_2, PtCDC20_4 and PtCDC20_5 had mistakes in the 3' region. This region contains the IR-tail sequence element (consensus IR) that occupies the $\mathrm{C}$ terminus of APC activators and the APC subunit APC10. Comparing with EST databases, all sequences were deduced and the IR-tail was identified (see Additional file 7). In PtCCS52A1_2 and OsCDC2O_2 genes, introns were incorrectly included. The opposite happened in the OsCDC20_1 gene, where one exon was absent. Alternative splicing variants were found in the Arabidopsis, rice and poplar APC/activator genes (data not shown) and could represent another layer of complexity in the organization and function of the APC. We selected only a single variant for further analysis.

Duplication of cell-cycle machinery components is rare in metazoans but it is a widespread phenomenon in plants [35-38].It has been proposed that duplication, followed by sequence divergence of promoter and/or coding regions, leads to novel and specialized functions unique to the plant kingdom [23,39]. Still, additional file 8 shows that duplications of individual subunits of the APC are not present in all plants; on the contrary, they may be restricted to one or to a small group of phylogenetically related plant species and may be involved in the organization of developmental events unique to this group.

\section{Chromosomal location of genes for APC subunits and activator}

The close similarity and presence of putative subunits and paralogs prompted us to investigate the genome distribution of plant APC/activators. APC genes were found in the five Arabidopsis chromosomes, 10 of the 12 rice chromosomes and 10 of the 19 poplar chromosomes (and seven in scaffolds) (see Additional file 9).

Only one APC subunit is duplicated in the Arabidopsis genome, AtCDC27a and $A t C D C 27 b$, and they are located on different chromosomes, 3 and 2 respectively; they share $47 \%$ identity and $64 \%$ protein similarity. A duplication event is predicted to have occurred in this case according to the tool Paralogons in Arabidopsis [40]. Arabidopsis CDC20 and CCS52 genes are located on chromosomes 4 and 5. AtCCS52A1 and AtCCS52A2 appear to be the result of a recent duplication and AtCDC20_1 and AtCDC20_2 are present in tandem on chromosome 4 [27].
The 19 rice APC and activator genes are distributed among all chromosomes, except chromosomes 10 and 11. Based on the sequence consensus, rice genome, segmental duplication information from the TIGR database was used to identify paralog genes. Each pair of paralogs located in the corresponding segmental duplication regions share high sequence similarity. Two subunits CDC23 and APC11 - are duplicated. OsCDC23_1 and OsCDC23_2 are located on chromosomes 2 and 6, respectively and share $92 \%$ identity. OsAPC11_1 and OsAPC11_2 are located on chromosome 3 and 7 , respectively and share $97 \%$ identify. No evidences for recent duplication events in rice activators were found.

In-silico chromosome mapping revealed that poplar APC/activators are scattered throughout the genome. Paralogs are also located on different chromosomes or scaffolds. It is possible, however, that some of the apparently closely related genes are in fact alleles from unassembled haplotypes, which are potential artifacts from shotgun assembly of this highly heterozygous genome. However, the apparent co-orthologs are divergent at the nucleotide level, as well as in the flanking gene order, and they are identified in the syntenic blocks which argue against the classification of the scaffold as a haplotype. Duplications of two APC subunits were identified; CDC27 and APC13. PtCDC27_1 is located on linkage group VIII and PtCDC27_2 on scaffold_211. Both are very similar to each other $-88 \%$ identity and $85 \%$ similarity in their amino-acid sequences, respectively. PtAPC13_1 is 95\% identical to PtAPC13_2 and they are located on linkage groups IV and XI, respectively. Poplar CCS52 genes are closely related, suggesting a recent genomic duplication event. Linkage groups III and I and VIII and X share large megabase-blocks in complete colinearity. PtCDC20_1 and PtCDC20_3 are located in chromosomal regions that might represent paralog segments - linkage groups XIII and XIX. Other CDC20 genes are located on linkage group XVI and scaffold_1538. The scattered distribution of APC/activators is in good agreement with previous studies that described large-scale duplication events in poplar [41].

\section{Phylogenetic analysis of APC TPRs subunits and activators proteins}

An important question is why the APC is composed of many different subunits, while most E3 ligases are composed of one to three subunits. Most of the APC subunits are conserved in all eukaryotes and remain tightly associated throughout the cell cycle $[42,43]$. It seems probable that the complexity of multiprotein domains is related to the intricate topology of APC $[44,45]$. One important domain in the APC subunits is TPR, which consists of 3-16 tandem repeats of 34 amino-acid residues. However, individual TPR domains can be 
dispersed in the protein sequence [46]. TPRs appear to act as versatile protein-protein interaction domains and it has been hypothesized that the TPR-containing proteins in the APC form a scaffold on which the other subunits assemble [47]. Five APC subunits contain TPR domains: CDC27, APC5, CDC16, APC7 and CDC23. TPR-containing sequences of primitive red algae Cyanidioschyzon merolae; green algae Volvox carteri, Chlorella sp., Micromonas sp and Ostreococcus sp; the bryophyte Physcomitrella patens; the licophyte Selaginella moellendorffii; the land plants Vitis vinifera and Sorghum bicolor; and the poplar, rice and Arabidopsis sequences were used to gain insight into the evolutionary relationship between land plants and algae. A phylogenetic tree was constructed with the MEGA4 neighbor-joining method, employing multiple alignments of 15 CDC27, 11 APC5, 12 CDC16, 9 APC7 and 14 CDC23 genes, with bootstrap analysis of 2,000 replicates to ensure statistical reliability (Figure 1 and Additional file 10). Subsequently, the 61 APC subunits were divided into five phylogenetic groups of ortholog genes. The cladogram obtained shows the OsCDC23 genes in the same branch, located in the same group as S. bicolor. Grapevine CDC23 genes are in the same group as poplar. The cladogram also shows that the poplar and grapevine genes are in the same branches in every group, and this could reveal slow evolutionary rates in woody when compared to herbaceous plants. On the other hand, while Arabidopsis CDC27a is in the same group of CDC27b, it is in a separate branch, and sequence divergence and evolution may reflect the accelerated life cycle of this plant. Interestingly, the APC5 and CDC16 clades do not contain duplicated genes. We have not identified an APC5 sequence in red algae $C$. merolae; however, this subunit sequence is not so well conserved among different organisms, and it is possible that the similarity is too low to be identified by BLAST comparison.

The APC7 gene is absent in green algae Chlorella $s p$ and $V$. carteri, as well as in the red algae $C$. merolae, but it is present in green algae Ostreococcus $s p$ and Micromonas sp. Chlorophytes (e.g., V. carteri) and Trebouxiophytes (e.g., Chlorella $s p$ ) have apparently lost the APC7 gene, but the Prasinophytes (e.g., Ostreococcus sp, Micromonas $s p$ ) and land plants retained this TPR subunit. These data also suggest that the primitive ancestor of Chlorophyceae and Trebouxiophyceae classes lost APC7 gene during green algae evolution (see Additional file 11). The APC7 gene is also not found in the yeasts, and it could mean that it is not required for APC function in unicellular organisms and it could be a recent duplication of another TPR subunit.

The Arabidopsis genome codes for six CDC20 genes and three CCS52 genes. In addition, CCS52 genes have been divided into two types ( $\mathrm{A}$ and $\mathrm{B}$ ) on the basis of functional and sequence analysis [27]. To gain an understanding of the evolutionary relationship between activators from land plants and algae, phylogenetic analysis was performed for activators from plants and algae genomes (Figure 2 and Additional file 10). We used EST sequences from Zea mays, Medicago truncatula and Saccharum officinarum to better estimate divergence between monocot and dicot sequences. The obtained phylogenetic tree reveals three major class groups. Three genes, $C m C D C 20, C m C C S 52$ and SmCDC20_3, are isolated from the others and thus need to be treated as nonconserved sequences. One clade groups all CDC20 sequences (except $C m C D C 20$ and SmCDC20_3), while two other clades included the CCS52A and CCS52B sequences (except CmCCS52). The CDC20 clade is divided into two subfamilies: one with $S$. moellendorffii and P. patens and another with algae and land plants. The number of CDC20 copies varies according to species. Arabidopsis has six copies, rice three, poplar five, grapevine four, sorghum three, S. moellendorffii three and P. patens four. The CCS52A clade contains more duplications than the CCS52B clade. All algae CCS52 genes, two copies of PtCCS52A, AtCCS52A and $S m C C S 52$, and three copies of PpCCS52 genes are grouped into the CCS52A branch.

Curiously, only one copy of the CDC20 and CCS52 (possibly a primitive A-type) genes was found in algae genomes. The absence of CCS52B type genes in algae, $P$. patens and $S$. moellendorffii is interesting because these species are at the base of plant evolution where divergence into A-type and B-type has not occurred yet. Like Arabidopsis, poplar has two copies of CCS52A; however poplar has an additional copy of CCS52B. Analyses of poplar gene evolution must take into account the most significant event in the recent evolution of the genus: a genome-wide duplication event at approximately 65Mya and is still detectable over approximately $92 \%$ of the genome [41]. Based on the age estimates of duplicate genes and homology microsynteny analyses, CDC20 and CCS52 gene pairs are represented within segmental duplication regions associated with the recent salicoid duplication event.

\section{Comparison of Arabidopsis, rice and poplar TPRs proteins} and determination of orthologous relationships

Progress in comparative genomics allows assessing the impact of gene and genome evolution on the appearance of novel biological functions and their effect on organismal complexity [48]. The importance of gene duplication in supplying raw genetic material for biological evolution has been recognized for decades and is still extensively studied. Gene duplication generates functional redundancy, which allows two identical genes resulting from the duplication to accumulate mutations 


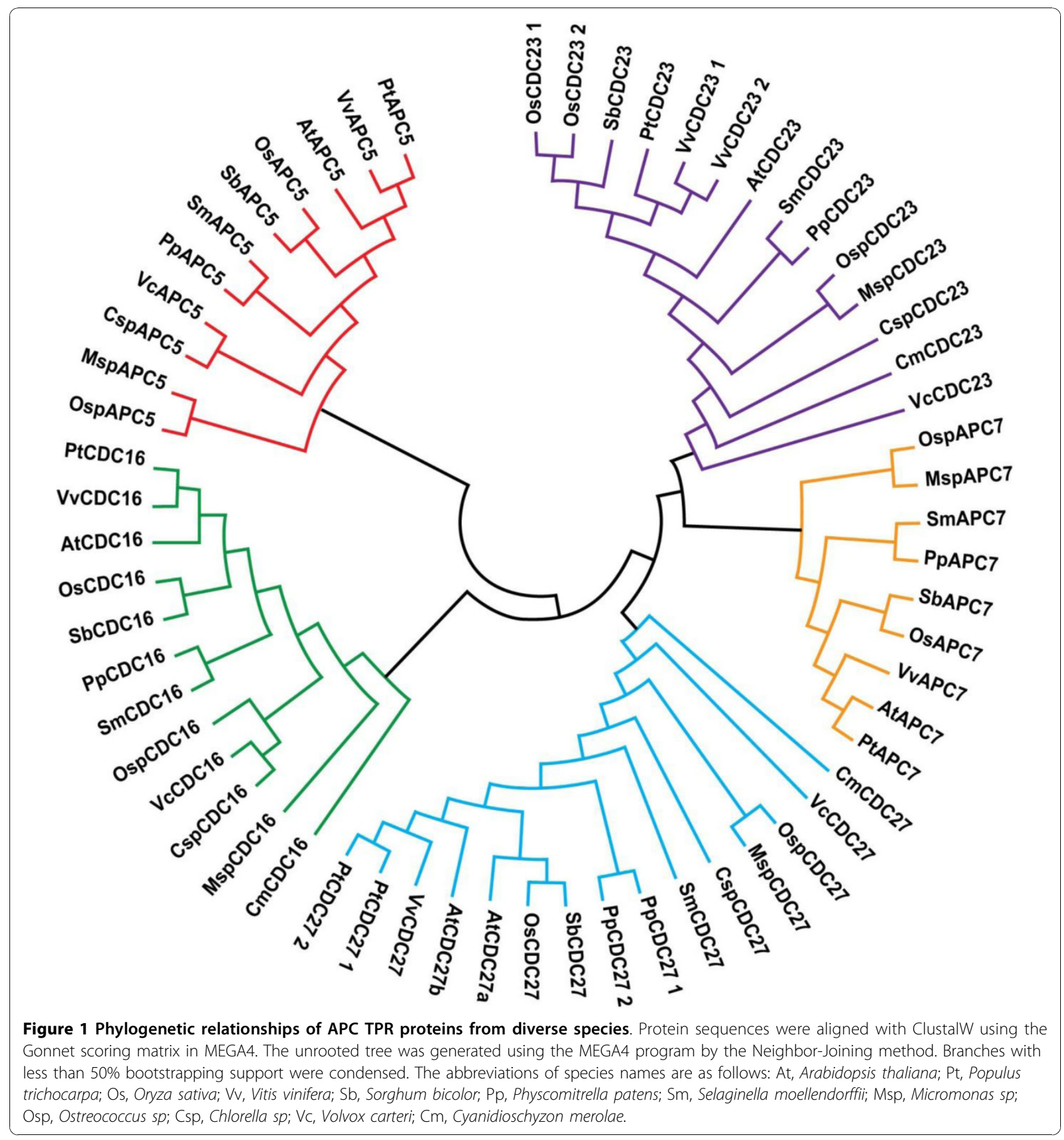

with relaxed selection pressure [49,50]. Differentiated or novel function may be achieved after a period of evolution. Segmental duplication (tandem duplication of a genomic segment) is the most prevalent way to generate redundant genes [51]. Segmental duplication can also happen on a smaller scale, resulting in duplicated exons, rather than an entire gene [52]. These segmental duplication blocks can create protein fragments (referred to as domain) that have structure and function [53].
The TPR domain consists of a 34-residue repeat that adopts a helix-turn-helix conformation, which is associated with protein-protein interactions [54]. The subunits CDC27, APC5, CDC16, APC7 and CDC23 have TPR domains, but the total number of TPR repeats and the position in each sequence is variable. Exon shuffling may be responsible for internal duplications in repeats and we decided to investigate the exon-intron organization of TPR genes in Arabidopsis, rice and poplar 


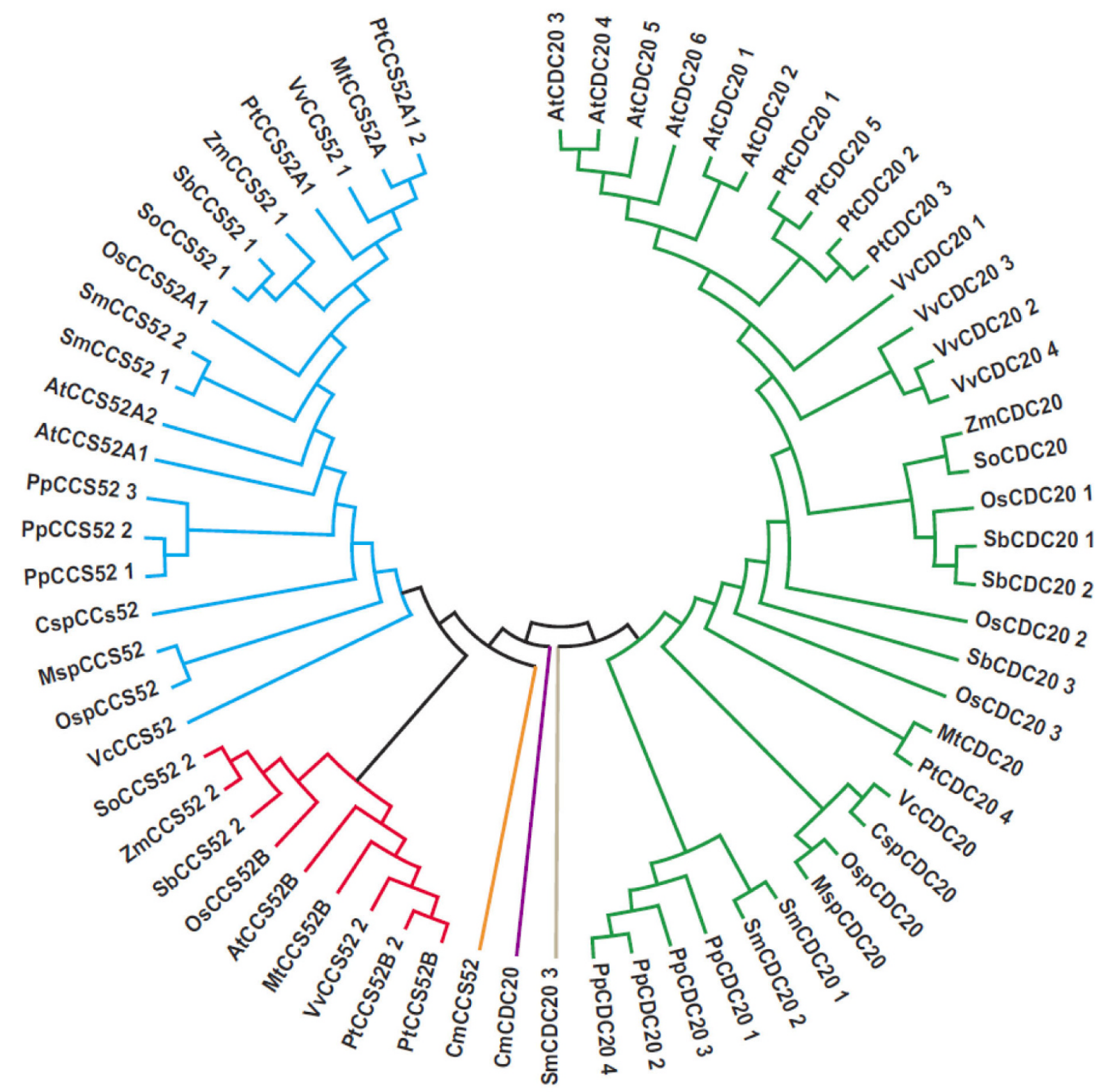

Figure 2 Phylogenetic relationships of CDC20 and CCS52 proteins from diverse species. Protein sequences were aligned with ClustalW using the Gonnet scoring matrix in MEGA4. The unrooted tree was generated using the MEGA4 program by the Neighbor-Joining method. Branches with less than $50 \%$ bootstrapping support were condensed. The abbreviations of species names are as follows: At, Arabidopsis thaliana; Pt, Populus trichocarpa; Os, Oryza sativa; Vv, Vitis vinifera; Zm, Zea mays; So, Saccharum officinarum; Sb, Sorghum bicolor; Mt, Medicago truncatula; Pp, Physcomitrella patens; Sm, Selaginella moellendorffi; Msp, Micromonas sp; Osp, Ostreococcus sp; Csp, Chlorella sp; Vc, Volvox carteri; Cm, Cyanidioschyzon merolae.

(Figure 3A) [55]. The number and position of exons and introns in the genomic sequences are remarkably conserved in some of the APC subunit genes. The CDC27 gene has two homologs in Arabidopsis and poplar, and only one in rice. Interestingly, the $\mathrm{CDC} 27$ gene maintains 16 exons in monocot and dicots. The APC5 and APC7 genes have one copy in all genomes and also maintain 20 and 18 exons respectively, in the three genomes. On the other hand, the $\mathrm{CDC} 23$ gene has two homologs in rice and only one in Arabidopsis and poplar, but the most significant information is the lower number of exons when compared with other TPR-containing genes. The OsCDC23_2 has 5 exons (exonintron annotation in Additional file 12) and $A t C D C 23$, PtCDC23 and OsCDC23_1 have 4 exons. Examining the CDC16 gene we identified 15 exons in poplar, 16 exons 

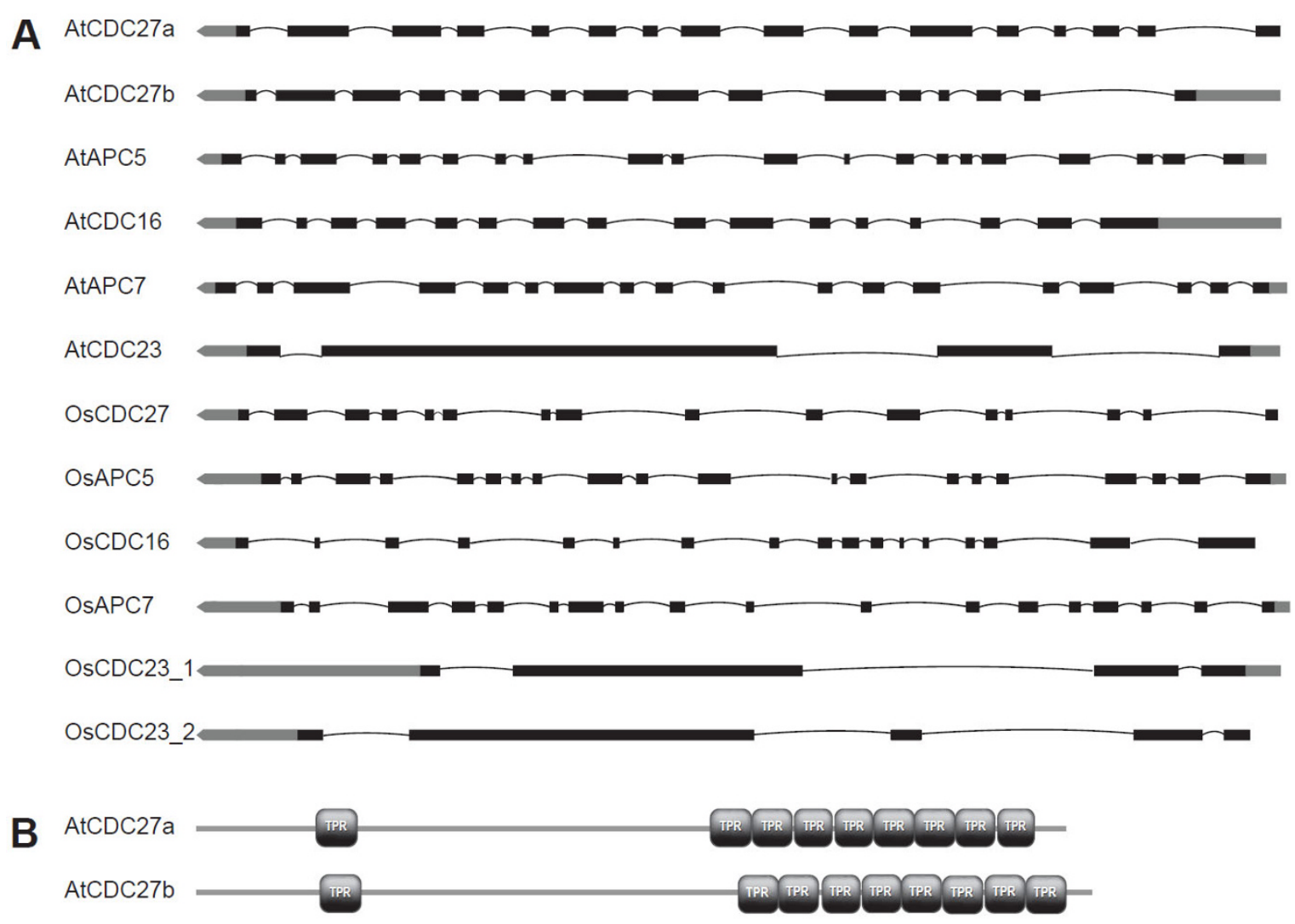

AtAPC5
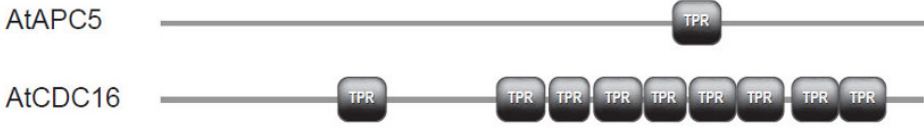

AtAPC7
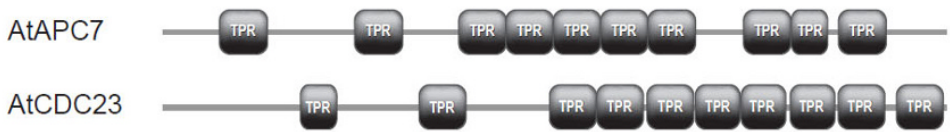

C

AtCDC27bxAtCDC16

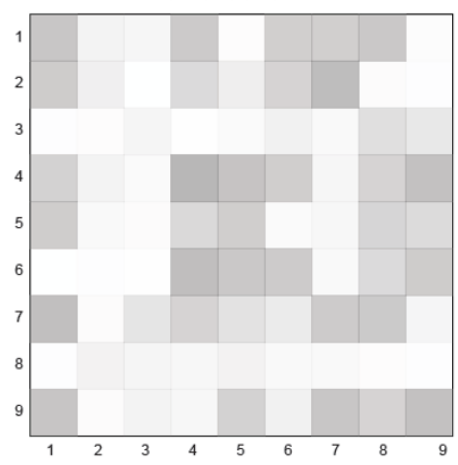

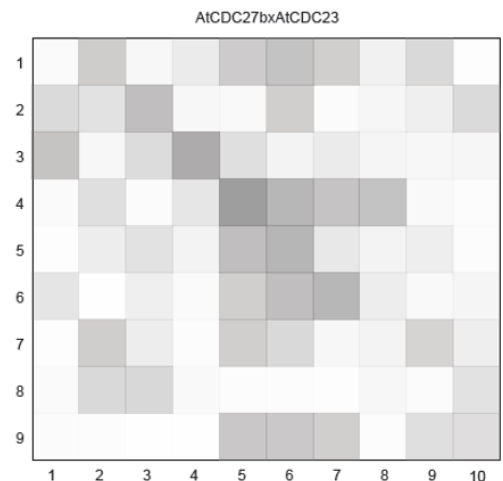

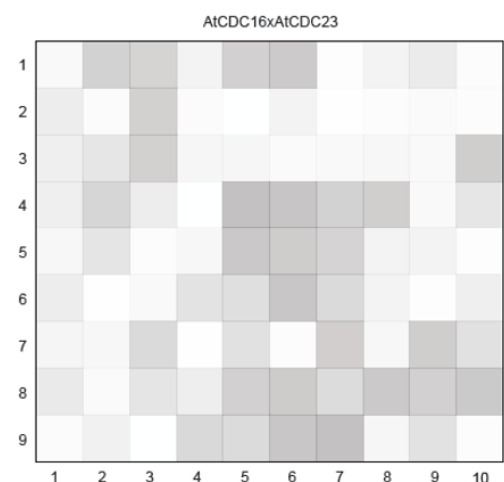

Figure 3 Analysis of Arabidopsis TPR subunits. A, Diagram of exon-intron structure of Arabidopsis and rice TPR subunit genes. Exons and introns are represented by black boxes and lines, respectively. Thick gray lines correspond to the untranslated regions. B, Schematic representation of TPR domains on Arabidopsis TPR subunit sequences. TPR domains and full-length proteins sequences are represented by black boxes and gray lines, respectively. C, Pattern of internal domain duplications in Arabidopsis TPR subunits. The intensity of shading reflects the alignment score, with a dark shading for higher scores. The numbers on each axis indicate the domains in N-to-C terminal orientation within the repeat. 
in Arabidopsis and 17 exons in rice. We next compared the exon organization of all TPR genes from Arabidopsis using domain prediction. Nine TPR repeat sequences were identified in AtCDC27a and AtCDC16 sequences, two in AtAPC5 and ten in the AtAPC7 and AtCDC23 sequences (Figure $3 \mathrm{~B}$ ). AtCDC27b has the same number of TPR domains as AtCDC27a. Tandem arrangement of TPR domains occurs predominantly at the C-terminal of AtCDC27a, AtCDC27b, AtCDC16, AtAPC7 and AtCDC23 sequences. AtAPC5 has only two TPR domains, one in the middle of the sequence and another at the C-terminal. The results show that TPR tandem repeat topology on protein sequences is at least partially related with the exon organization of TPR subunit genes.

In the APC subunits, the tandem arrangement of TPR domains suggests that the elements evolved by duplication followed by primary sequence divergence. Because TPR are conserved structure repeat elements which are often divergent at the primary sequence level, TPR domain sequences can diverge a great deal over time and have poor consensus motifs. An approach to investigate the evolution of TPR domains in the subunits is to identify patterns of TPR duplication from the alignments. Distinct patterns of repetition can often be distinguished, and we tested whether the Arabidopsis TPR proteins have defined internal repetition blocks. The sequences of TPR proteins were aligned to each other or in pairs using the methodology described in [56]. The pairwise sequence similarities between all repeating domains in a protein were examined using the SmithWaterman algorithm to identify patterns of duplication from the alignments [57]. The alignment scores between the domains were displayed in a matrix (Figure $3 \mathrm{C}$ and in Additional file 13). Comparing the proteins to themselves, there are similarities among domains 4-6 in AtCDC27; and among domains 5-9 in AtCDC16. AtAPC7 has a pattern for domains 1-2, AtCDC23 has patterns of similarities for domains 6-8; and no pattern was found to for AtAPC5. Comparing the proteins in pairs, we can observe a diagonal match between AtCDC27 domains 2-6 and AtCDC23 domains 3-7. There are also indications of similarities for the pairs AtCDC27 and AtCDC16, AtCDC16 and AtCDC23. Although there are no unambiguous duplication patterns for any of the proteins, the results suggest that TPR domains in the APC genes have evolved by duplication of the central elements, or that higher constraints are imposed to changes in the primary sequence of these repeats.

Expression analysis of APC subunits and activators genes Gene duplication can be a source of innovation for the increased developmental complexity of plants.
Expression patterns can provide important clues for gene function under specific conditions. We examined the expression of the APC subunit and activators genes in 5-day old rice roots and shoots, and in sheath and blade of mature tissues (Figure 4A). As expected, there are higher levels of gene expression in tissues with high proliferation rates, although there is more mRNA in shoots than in roots. However, a complex pattern is observed when their expression is examined in mature blade. In general, there is a general decrease in expression for several genes, as expected. However, the degree of reduction is variable. The mRNA levels of OsAPC1, OsAPC2, OsAPC4, OsAPC5, OsCDC16, OsCDC23_2, OsAPC10, OsAPC11_2, OsCDC26, and OsAPC13 are reduced in both sheath and blade compared to aerial part. On the other hand, OsAPC11_2 and OsCDC27 mRNA levels are reduced only in the sheath, but not in the blade. Finally, there is no reduction of OsAPC7 mRNA levels in both sheath and blade. In contrast, expression levels of activator genes are markedly reduced in mature leaves. However, although CCS52A mRNA levels are lower in both sheath and blade, the reduction is less pronounced. In Medicago, the CCS52A gene has been implicated in control of endoreduplication in nodules [58]. Recently, it has been shown that the CCS52A gene is important for maintenance of meristem activity in roots of Arabidopsis [59]. Therefore, it is possible that the CCS52A could form a specialized complex with some of the APC subunits and assume a unique function in mature leaves.

Growth response to dark is part of an integrated developmental change throughout all the plant organs [60]. In Arabidopsis, hypocotyl cells undergo up to two rounds of endoreduplication in light-grown seedlings, whereas an additional round can be observed specifically during dark-grown development $[61,62]$. The endoreduplication regulatory mechanism is thought to be common to the $G_{1}-S$ transition of the mitotic cell cycle [63]. $\mathrm{G}_{1}-\mathrm{S}$ transition is important for CDK inactivation by cyclin proteolysis, mediated by $\mathrm{APC}^{\mathrm{CDH} 1}$, which then maintains cyclin instability in $G_{1}$ and enables a new round of DNA replication by allowing the assembly of pre-replication complex (pre-RCs) [64]. Almost all rice APC subunits have similar expression patterns, both in roots and shoots (Figure 4B). However, OsAPC11_1 and OsCDC26 mRNA levels increased in darkness. Interestingly, the two OsCDC23 genes are differentially expressed in etiolated roots. While OsCDC23_1 mRNA levels did not change in roots grown in the dark or light, OsCDC23_2 mRNA levels in roots were much lower when rice plants were grown in the dark. Similarly, in etiolated roots OsAPC11_1 mRNA levels increased while OsAPC11_2 levels decreased. These results could indicate that, following gene duplication, 


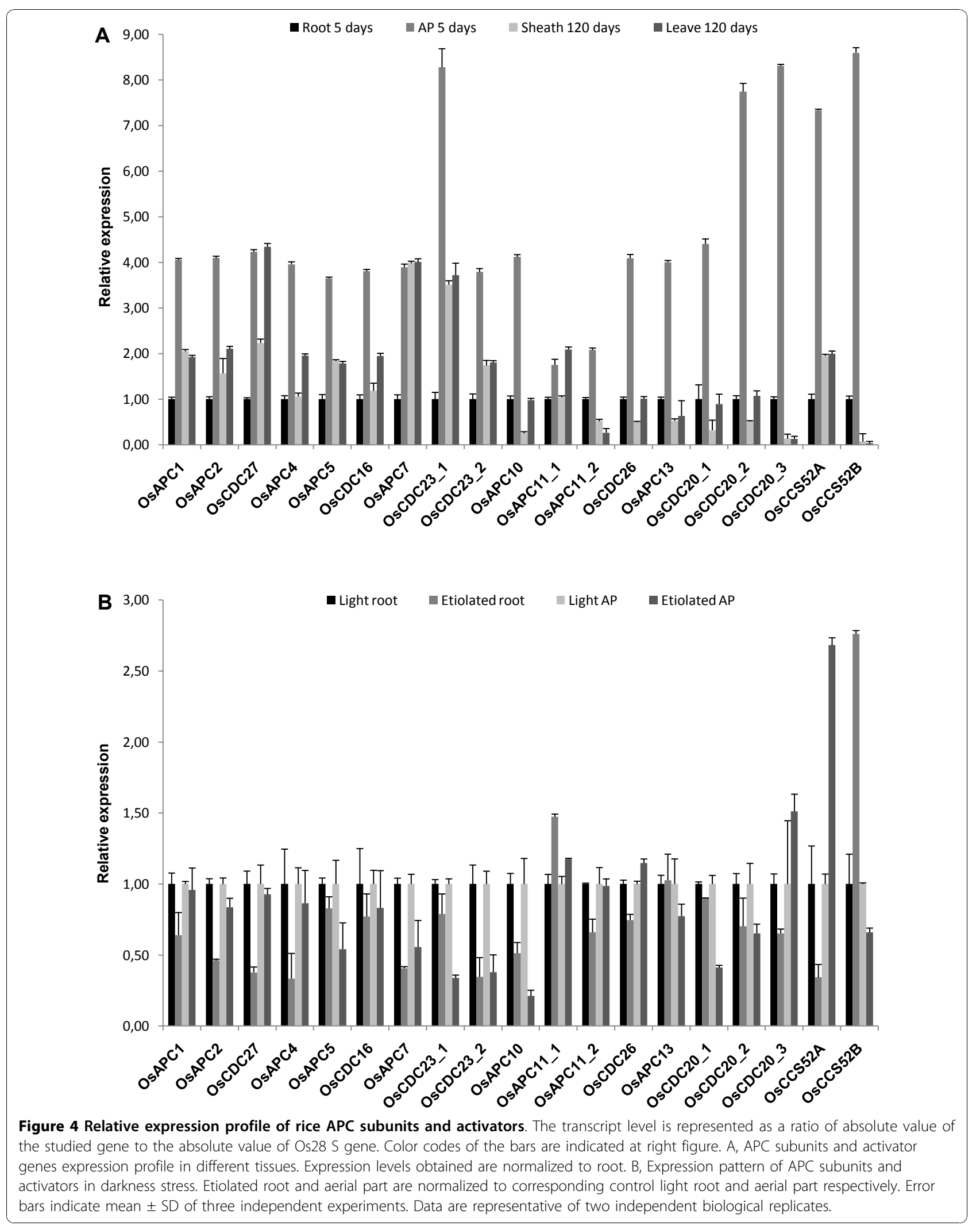


the APC11 and CDC23 genes are assuming new functions in rice.

The activators OsCCS52A and OsCCS52B exhibited high levels of mRNA in darkness and OsCDC20_3 showed a lower level. One hypothesis for the high mRNA levels of CCS52 genes is that the $\mathrm{APC}^{\mathrm{CCS} 52}$ acts as a negative regulator of CYCA2;3/CDKA;1 complex in Arabidopsis [65]. The CYCA2;3/CDKA;1 complex acts as a negative regulator of endocycle and the APC activation by CCS52 may increase CYCA2;3 degradation and consequently the progression of endocycle in darkness [65].

To investigate the expression of the APC genes and activators during rice seed development, we examined the expression of the APC subunits and activators in endosperm, pericarp and inflorescence tissues (Figure $5 \mathrm{~A})$. Expression levels were measured in the inflorescence at reproductive stage 3 (before anthesis, 70 days after emergency), pericarp and endosperm at reproductive stage 6 (milk stage, 90 days after emergency). Levels of mRNA from all APC subunits were higher in the endosperm, except OsAPC1 and OsAPC5, which were present in higher amounts in the inflorescence. The duplicated $O s C D C 23$ genes displayed similar expression profiles in the seed tissues examined. However, the OsAPC11 gene seems to be differentially expressed; OsAPC11_2 showed reduced amounts of mRNA in the inflorescence when compared to the pericarp and endosperm. Using the Genevestigator Analysis tool, we compared the expression levels of the APC genes with qRTPCR results (see Additional file 14) [66]. Although only the genes OsAPC2, OsAPC7, OsAPC10 and OsAPC13 are present in the microarray slides, their expression is similar to what we observed in this work.

The expression patterns of the various APC activator genes were analyzed. The OsCCS52B transcript was expressed at low levels in the inflorescence and OsCDC2O_1 was barely detected in the same tissue. Interestingly, transcripts for OsCDC2O_2 and OsCDC20_3 were detected in inflorescence, a tissue with a high rate of proliferation, suggesting their involvement in mitotic activities [67]. The OsCCS52A had high levels in the endosperm and this may be explained by the fact that the transition from a mitotic to an endocycle in endosperm cells involves a spike in activity of the master cell cycle regulators belonging to the CDK family and APC complex, which subsequently decreases as endocycle progresses [68]. These contrasting expression patterns for OsCCS52 and OsCDC20 could suggest distinct functions for these APC activators during seed development.

Based on the direct relationship between the increase of auxin and cellular proliferation we treated rice seedlings with naphthaleneacetic acid (Figure 5B). After treatment for 2 and 8 hours, all APC and activator genes increased their expression. This result indicates that the rice APC subunits and activators might be involved in the regulation of the cell division events triggered by auxin in the seedlings.

We analyzed the expression in leaves of PtAPC10, PtCDC27_1, PtCDC20_1, PtCCS52A1_1 and PtCCS52B genes by qRT-PCR (see Additional file 2). Interestingly, PtCCS52B exhibited lower mRNA levels when compared to PtCCS52A1_1. Recent data suggest that the $M t C C S 52 B$ may have specific roles in M-phase progression, alternative or complementary to those of MtCCS52A [69]. Thus, CCS52A might have major roles in post-mitotic, differentiating cells, in which degradation of specific APC targets could contribute to differentiation of given cell types, tissues, or organs [70].

\section{Conclusions}

Through genome-wide bioinformatics analyses of the complete genomes of Arabidopsis, rice and poplar, we identified ortholog genes of APC subunits and activators. In addition, their occurrence was demonstrated in algae, bryophyte and other monocotyledonous and dicotyledonous species as well. A distinctive feature of these genomes is the presence of characteristic duplication patterns (see Additional file 8). In Arabidopsis, only the CDC27 gene is duplicated. Rice and poplar have two duplicated subunits, CDC23-APC11 and CDC27-APC13 respectively. Among other plant genomes, grape and $P$. patens have two copies of the CDC23 and CDC27 genes, respectively, and grape has two copies of the APC13 gene. The two isoforms of CDC23 and APC11 genes in rice are differentially expressed in light-dark grown plants and one of APC11 subunits is also more expressed in the inflorescence than the other. In Arabidopsis, the duplicated copies of the $\mathrm{CDC} 27$ gene exhibit significant sequence differences, and seem to have assumed specialized functions, indicative of the occurrence of APC subcomplexes [23]. While the coding sequences of the rice genes are quite conserved, it is possible that their controlling elements have diverged and that they are assuming new functions in the APC during plant development. Paralogs usually display different functions, whereas orthologs may retain the same function [71]. We tested the frequency of $\mathrm{Ka} / \mathrm{Ks}$ for CDC27, CDC23, APC11 and activators orthologous genes and found interesting values (see Additional file 15). The CDC23 and CDC27 TPR genes had the highest values (greater evolutionary pressure) and the activators and APC11 subunit lower. These results reveal the importance of maintenance of the amino acids conserved in RING finger proteins and the adapters. Analyses of the promoters of the rice subunit genes revealed different regulatory sites for duplicated genes, suggesting different regulation of their expression (see Additional 


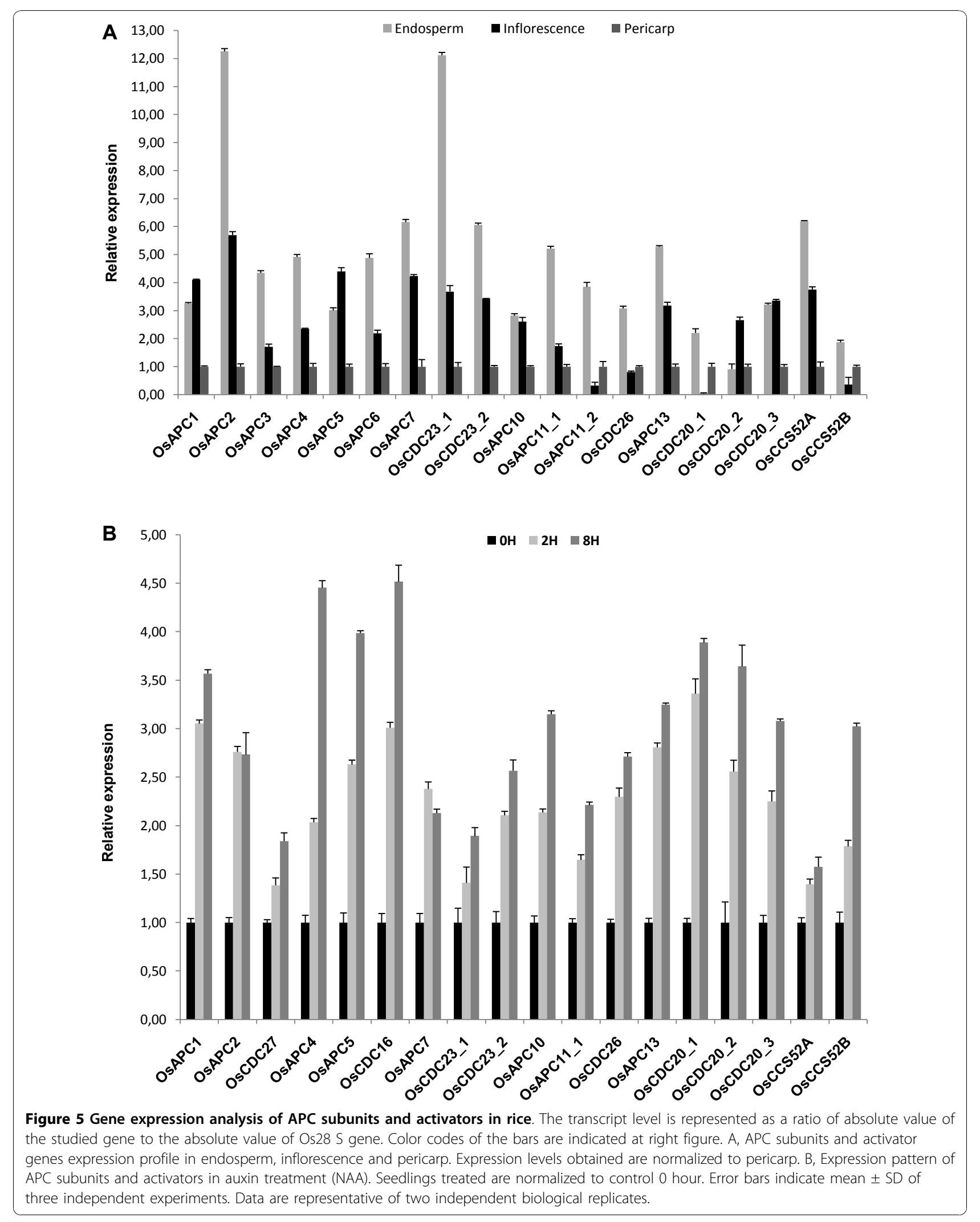


file 16). As expected, the most common cis-acting elements in all genes analyzed have functions related to cell division. Strikingly, OsCDC23 and OsCDC23_1 and OsCDC23_2 do not show any cis element in common and only two cis-regulatory elements are found in both OsAPC11_1 and OsAPC11_2. However, the OsAPC11 and $O s C D C 23$ gene sequences present very little variation across taxa, possibly due to strong purifying selection.

As in yeasts, APC7 is not present in the green algae Chlamydomonas reinhardtii, V. carteri and Chlorella spp. The absence of this subunit at the root of animal and land-plant evolution suggests that this subunit may have appeared after a gene duplication event of a TPRcontaining subunit gene, and it may not be essential for APC activity in unicellular organisms. Curiously, it might be the case also in later metazoans, once Drosophila APC7 knockout mutant flies and null mutants are viable and fertile [72].

The activators CDC20 and CCS52 have many copies in plants. This is in sharp contrast with the situation in metazoans, where only one copy of each regulator is present per genome. Due to the duplications, Arabidopsis contains three CCS52 activators; CCS52A, CCS52A1 and CCS52B. In addition, six CDC20 genes were found, but AtCDC20_6 is probably a pseudogene because the important motif KEN-box is absent [12,73,74]. In poplar, the CCS52A and CCS52B genes are also duplicated. Five CDC20 genes are present in poplar, the same number found in Arabidopsis. Interestingly, rice contains one copy of the CCS52A and CCS52B genes; and three copies of $\mathrm{CDC} 20$ genes. This suggests that the duplication of the CCS52 genes occurred in dicotyledonous plants after the separation from monocotyledons. Recent duplication of the CDC20 genes has occurred also in Arabidopsis and poplar.

Plants can adopt dramatically different alternative developmental pathways and must integrate cell-cycle progression, growth and development in response to environmental cues. It is believed that multiple members of a specific gene family of a particular organism are the natural products generated from the long evolutionary history that the organism experienced [75]. The number of members of a gene family reflects a succession of genomic rearrangements and expansions due to extensive duplication and diversification that occurred in the course of evolution. Phylogenetic analysis of the APC subunits and activator proteins is very illuminating. The fact that all subunit types except APC7, were found in all plant genomes analyzed, including red algae, green algae and other primitive organisms, suggests that the complete set of genes encoding the APC subunits was already present in the common ancestor of plants and animals. The activators descend from a common ancestor and correspond to well-conserved structures. In all algae species analyzed, there is only one copy of CDC20 and CCS52 genes. Land plants have amplified the number of activators and this fact may be associated with substrate specificity and or with the complexity of their developmental programs.

By comparing the similarity between different TPR domains within one protein it might be possible to trace the evolutionary history of the internal duplications. The alignment scores between domains showed limited similarities among TPR subunits of $A t C D C 27, A t C D C 16$, AtAPC7, AtCDC23 and AtAPC5. The sequence of TPR subunits can diverge a great deal over time, limiting their consensus motifs and making it difficult to detect unambiguous duplication patterns for any of the proteins. Nevertheless, pairwise comparisons indicated greater similarity for the central domains, especially between $\mathrm{CDC} 27$ and $\mathrm{CDC} 23, \mathrm{CDC} 27$ and CDC16, and CDC23 and CDC16. In all cases, higher similarity scores between TPR subunits 4 in CDC16 and CDC27, and TPR subunit 5 in CDC23 were detected, suggesting that the TPR tandem evolution most likely started from the middle domains. Because the primary amino acid sequence of TPR subunits tends to diverge, the higher degree of similarity among the central subunits may also indicate that stronger evolutionary constraints are applied to these regions.

Previously, we have shown that the Arabidopsis subunit genes are differentially expressed according to whether the plants are grown in the dark or under light. When Arabidopsis plants were grown in the dark, only AtAPC1, AtAPC2 and AtCDC16 increased mRNA levels [21]. However, qRT-PCR expression profiles of rice APC and activator genes showed spatial modulation of gene expression of the duplicated subunit genes in different tissues (root and aerial parts) of plants grown under different light treatment. OsAPC11_1 mRNA accumulates to higher levels in etiolated roots when compared with OsAPC11_2 mRNA, and OsCDC23_1 mRNA is present in etiolated roots at almost twice the levels of OsCDC23_2. Likewise, the rice OsAPC11 genes are differentially expressed in the inflorescence, with activators that have very complex expression patterns. Altogether, these data suggest that the duplicated rice APC subunits and activators could participate in different APC subcomplexes and that they may have assumed new specialized functions as they diverged during evolution.

Most E3 ligases are composed by one or two polypeptides [76]. In contrast, the cell cycle regulators SCF and APC are formed by a larger number of subunits. However, for both complexes, biochemical activity can be achieved by only two subunits, a Cullin related protein together with a RING finger protein. In both cases, substrate specificity is defined by the adaptor subunit. The 
human genome contains 69 F-Box proteins - the SCF activator - and two APC activators, CDH1 and CDC20 $[77,78]$. In plants, SCF-related complexes are involved in many physiological processes and approximately 700 F-box proteins have been identified in Arabidopsis [79]. The number of APC activators have also suffered an expansion in the higher plant lineages, though a more modest one, the number varying from nine in poplar and Arabidopsis to six in rice. We and others have shown that differential expression of APC subunits could be a source of complexity in regulation of the APC $[21,23]$. The results presented here suggest that gene duplication, followed by sequence divergence and differential expression, could be another tier of regulation of ubiquitin-mediated proteolysis mediated by specific APC complexes composed by particular subunit isoforms. Future studies employing functional genomics approaches will be required to define the impact of duplications or splicing variants on cell-cycle progression at the cellular level and the associated plant developmental processes at the whole-organism level.

\section{Methods}

\section{Gene Identification and Chromosomal Location}

Searching of multiple databases was performed to find members of APC, CDC20 and CCS52 in Arabidopsis, rice and polar. The strategy to obtain each gene in a genome was the following; Arabidopsis sequences have been published [80], but additional searches were carried out against the TAIR8 database http://www.arabidopsis.org. The sequences obtained were then used as queries to search against the following databases: Oryza sativa L. cv. Nipponbare - The Institute for Genomic Research (TIGR) http://rice.plantbiology.msu.edu, Populus trichocarpa Nisqually-1 - DOE Join Genome Institute http://genome.jgi-psf.org/Poptr1_1/Poptr1_1.home. html, Sorghum bicolor BTx623 - DOE Join Genome Institute http://genome.jgi-psf.org/Sorbi1/Sorbi1.home. html, Vitis Vinifera PN40024 - Genoscope http://www. genoscope.cns.fr/externe/GenomeBrowser/Vitis/, Ostreococcus $s p$ RCC809 - DOE Join Genome Institute http:// genome.jgi-psf.org/OstRCC809_1/OstRCC809_1.home. html, Volvox carteri - DOE Join Genome Institute http://genome.jgi-psf.org/Volca1/Volca1.home.html, Chlorella sp. NC64A - DOE Join Genome Institute http://genome.jgi-psf.org/ChlNC64A_1/ChlNC64A_1. home.htmlMicromonas sp NOUM17 (RCC299) - DOE Join Genome Institute http://genome.jgi-psf.org/MicpuN3/MicpuN3.home.html, Physcomitrella patens DOE Join Genome Institute http://genome.jgi-psf.org/ Phypa1_1/Phypa1_1.home.html, Selaginella moellendorffii - DOE Join Genome Institute http://genome.jgi-psf. org/Selmo1/Selmo1.home.html and Cyanidioschyzon merolae http://merolae.biol.s.u-tokyo.ac.jp databases using the BLASTP and TBLASTN programs. In addition, Arabidopsis APC and activator sequences were used to search against Zea mays, Medicago truncatula and Saccharum officinarum EST databases at Gene Index DFCI http://compbio.dfci.harvard.edu/tgi/cgi-bin/ tgi/Blast/index.cgi. Sequences with significant similarity to Arabidopsis proteins were downloaded into our database and annotated accordingly. Sequence conservation analysis of APC and activators genes was conducted using the BLAST tool with default curve calculation parameters; amino acids sequence "conservation similarity" of $50 \%$. Chromosomal locations of APC subunits and CDC20/CCS52 genes were obtained using the BLAST server and additional physical localization tools of each genomic browser.

\section{Identification of Protein Domains}

The SMART database http://smart.embl-heidelberg.de was used to confirm whether each predicted proteins sequence maintains conserved domains and motifs. The TPRpred http://toolkit.tuebingen.mpg.de/tprpred search program was used to confirm the TPR domain in specific sequences [81].

\section{Phylogenetic Analysis}

Phylogenetic analysis of TPR subunits and activators CDC20 and CCS52 was carried out using the NeighborJoining method in the Molecular Evolutionary Genetics Analysis software package - MEGA4 [82]. Alignment for tree construction was done using ClustalW with the Gonnet scoring matrix. Reliability of the obtained trees was tested using bootstrapping with 2,000 replicates.

\section{Exon-intron and TPR Domain}

Exon-intron information was obtained from TAIR, TIGR and JGI databases. The sequences of the repeating domains were extract and aligned to each other using the Smith-Waterman alignment tool in the EMBOSS package and default parameters $[56,57]$. This gave pairwise alignment scores between all individual domains in a repeat.

\section{Plant Materials}

Rice seeds (Oryza sativa L. cv. Nipponbare) were disinfected with $5 \%$ sodium hypochlorite for $20 \mathrm{~min}$ and thoroughly washed with water. For darkness treatment, seedlings were grown hydroponically $(0.5 \mathrm{X}$ Hoagland's solution) in a greenhouse at $25-28^{\circ} \mathrm{C}$ for 2 weeks. The control group was grown with a photoperiod of $12 \mathrm{~h}$ light/12 $\mathrm{h}$ dark and the etiolated group was grown in darkness. For auxin treatment, seedlings were grown in Petri dishes (0.5X Hoagland's solution agar) in the greenhouse at $25-28^{\circ} \mathrm{C}$ for 2 weeks. Seedlings were transferred to new Petri dishes and incubated for 2 and 
$8 \mathrm{~h}$ in either water or $2 \mu \mathrm{M}$ NAA solution. Harvested seedlings were frozen in liquid nitrogen immediately and store at $-70^{\circ} \mathrm{C}$ until RNA isolation. Leaf poplar RNA was provided by Rodrigo T. Lourenço (Forest Biotechnology Group, North Carolina State University).

\section{Promoter analysis and significance calculations}

To identify promoter regions of APC subunits, $1000 \mathrm{bp}$ of sequence preceding each annotated gene from RAPDB database were extracted manually. These putative promoter sequences begin immediately upstream of the 5 ' UTR for transcription units with an annotated 5' UTR. To identify overrepresented promoter elements, the putative promoter regions upstream of APC subunits were analyzed. Known plant promoter elements and their annotation were downloaded from PLACE (A Database of Plant Cis-acting Regulatory DNA Elements) http://www.dna.affrc.go.jp/PLACE/[83]. For each promoter, the null distribution for each PLACE motif was modeled by counting the number of occurrences for each word within each of all rice gene promoters $(1,000$ surrogates) according to [84]. This approach provided an opportunity to determine the level of consistency between the observed frequencies in a single promoter motif with that across all other sequenced gene promoters. A one-tailed $p$ value was estimated for each motif based on the $\mathrm{Z}$ score of the difference of the actual word count for each promoter (Ctrue) minus the mean count from the 1,000 surrogates (Csurr) relative to standard deviation (SD) from the 1,000 surrogates (SDsurr) [i.e., $\mathrm{Z}=$ (Ctrue_Csurr)/SDsurr]. For each motif the $\mathrm{p}$ value calculated was the probability to the right of the observed count calculated on the null distribution from all promoters from the genome. If this probability was less than $5 \%$, the motif was considered significantly overrepresented. These calculations were implemented using Delphi scripts.

\section{Data Analysis for Ka/Ks}

The ratio of nonsynonimous to synonymous nucleotide substitution rates $(\mathrm{Ka} / \mathrm{Ks})$ was calculated as described by Siltberg and Liberles [85]

\section{Gene Expression Analysis}

Oryza sativa japonica subspecies was used to prepare all generic material. Total RNA was extract from materials according to [86]. After treatment with RNAse-free DNase I $(0.5 \mathrm{u} / \mu \mathrm{g}$ RNA), total RNA $(2.5 \mu \mathrm{g})$ was transcribed using random hexamer primers according to the manufacturer's protocol (Applied Biosystems). The cDNA was amplified using Taqman ${ }^{\circ}$ Reverse Transcription Reagent kit (Perkin-Elmer Applied Biosystem) on the GeneAmp 9700 thermocycler (Applied Biosystems) under standard conditions. Transcript levels were determined by qRT-PCR using a 7500 Real-Time PCR System (Applied Biosystems). For poplar gene expression analysis, cDNA synthesis and qRT-PCR were performed as described for rice. The data were first normalized to the level of expression of Os28 S, OsActin and PtActin for each RNA sample. Primers used for real-time RT-PCR were designed in gene specific region using Primer Express V3.0. Gene primer sequences used in the qRT-PCR analysis are listed: Os28 S forward (F) 5' GCGAAGCCAGAGGAAACT 3', Os28 S reverse (R) 5'GACGAACGATTTGCACGTC 3', OsActin F 5' CTTCATAGGAATGGAAGCTGCGGGTA 3', OsActin R 5' CGACCACCTTGATCTTCATGCTGCTA 3', OsAPC1F 5' CTTGAGCTCTGCTTGCATCT 3', OsAPC1 R 5' GCTTACAGCCATCTGCAGTC 3', OsAPC2 F 5' AATGCTGGGGACAATCTTCT 3', OsAPC2 R 5' TAATGGGTCTGCTTCCACAG 3', OsCDC27 F 5' AGCGACTTGCTACCTTCACA 3', OsCDC27 R 5' TTGACAGGACACAAGGCTTC 3', OsAPC4 F 5' CGACAAGGATGGCCTGTTAT 3', OsAPC4 R 5' GAAGCGCTTGAAAATTCCTG 3', OsAPC5 F 5' TTCGTTGGTCTATGCAACCT 3', OsAPC5 R 5' AACGGGAACTTCTCTTCAGC 3', OsCDC16 F 5' ACAGGAGGAGGGTGATCAAG 3', OsCDC16 R 5' GATTTTGCTTGCGTGAAGAA 3', OsAPC7 F 5' AGACTTCAGGGGAGCTCAAG 3', OsAPC7 R 5' TTGAGAGCTTTTGCAGACTGA 3', OsCDC23_1 F 5' CGCTGAAGCTTAATCGAAAGT 3', OsCDC23_2 R 5' GACCAAGACCATACCAAGCA 3', OsCDC23_2 F 5' CAGTTCTGGTGGAATCTGTCA 3', OsCDC23_2 R 5' CAAGATGTGCACTAGCAAGGA 3', OsAPC10 F 5' TGATCCCCGAGAAACATTC 3', OsAPC10 R 5' GAAGTGAAGTGAAATGGCTGAT 3', OsAPC11_1 F 5' CCAGGATGAAACCTGTGGTA 3', OsAPC11_1 R 5' GTTTGAGAATTGACCCACTTGA 3', OsAPC11_2 F 5' AAACATGCGGCATATGCA 3', OsAPC11_2 R 5' AAGTGGCGTAGATGTCTGAGAA 3', OsCDC26 F 5' ATCGGCCTCCCTACCAT 3', OsCDC26 R 5' GAGGAGAGGCTAGGGTTTGG 3', OsAPC13 F 5' TTGTGCTGGTTGGCTTTC 3', OsAPC13 R 5' CGTCCTCATCGACATCGT 3', OsCDC20_1 F 5' ATGATCGGTGCATCAGGTT 3', OsCDC20_1 R 5' GGCAAAGAACACGAGCAGT 3', OsCDC20_2 F 5' CCTGTCCGGAATAAACCTGT 3', OsCDC20_2 R 5' CACTCGATCTCATCGGAGAA 3', OsCDC20_3 F 5' TTCACATTTGGGATGTGTCC 3', OsCDC20_3 R 5' TACCACCACCTCCAGTTGC 3', OsCCS52A F 5' GCCCCAGGAAGATCCCTA 3', OsCCS52A R 5' TGCTGCATGCATTCCATAA 3', OsCCS52B F 5' GTCACCAAGCTCTGCGATT 3', OsCCS52B R 5' TCCTCCCATGTTCCTAATCC 3', PtActin F 5' GGTCAagGCTGGgTTtGCT 3', PtActin R 5' TCGCCAACATAGGCATCTTTT 3', PtAPC10 F 5' atgacCCTAgggaAaCATTt 3', PtapC10 R 5' 
AAAAGGCTGATGCGGAAAAG 3', PtCDC27_1 F 5' TGGCCTTCCAAACCTGTCAT 3', PtCDC27_1 R 5' GGCATGCTAAgATtgGAaCCA 3', PtCDC27_2 F 5' AGCCGGATGTTATTTGCAAAA 3', PtCDC27_2 R 5' ATGCCGCTTCAGCTTCATTT 3', PtCDC20_1 F 5' TTTATTCCAAACCGGTCAGC 3', PtCDC20_2 R 5' TCGGTTCATGTTCAAGGATTC 3', PtCCS52A F 5' TTCCCCCTCGAGACCTATTT 3', PtCCS52A R 5' CATtggGatTGTCCTCCTTC 3', PtCCS52B F 5' CGTATCATCACCCAGAGCAA 3', PtCCS52B R 5' TCGTTGCCTCCTTCTTTAACA 3'. Data were analyzed using 7500 SDS software V1.4 (Applied Biosystems).

\section{Additional material}

Additional file 1: Text file containing amino acid sequences in FASTA format for APC and activators genes used in these analyses.

Additional file 2: Confirmation of functionality of Poplar PtCDC27_2. A, sequencing of genomic DNA corresponding to PtCDC27_2 locus. Red box shows the actual triplet. B, Real-time PCR detection of PtCDC27_2 mRNA, as well of PtCDC27_1, PtCDC20_1, PtAPC10, PtCCS52A1_1 and PtCCS52B.

Additional file 3: Gene Index (partial) sequence of OsAPC1. An EST was recovered from the Gene Index Project, showing part of (upper). The coding region was underlined and stop codon was marked in red. This sequence was translated in silico (lower).

Additional file 4: Fragments from 5'region of PtAPC5 and fragments from the $3^{\prime}$ regions of genes PtAPC4 and PtAPC5 were found in the EST database. The additional sequence of OSAPC4 (not present in Arabidopsis nor poplar), product of incorporation of an intron, is boxed in yellow. Differences in 5'region of OSAPC5 are also highlighted in yellow.

Additional file 5: Comparison of 5'region of CDC16 and APC11_2 genes between Arabidopsis, poplar and rice. The additional sequences are boxed in yellow.

Additional file 6: Re-sequencing of 5'region of AtCDC20_6.

Additional file 7: Correction of APC activator sequences. Gray boxes: The conserved APC interacting motifs C-box, CSM, IR-tail, CBM. Differences are boxed in yellow.

Additional file 8: APC subunits in plant genomes and red algae. $\mathrm{Cr}$, Chlamydomonas reinhardtii; Vc, Volvox carteri; Csp, Chlorella sp; Msp, Micromonas sp; Osp, Ostreococcus sp; Cm, Cyanidioschyzon merolae; Pp, Physcomitrella patens; Sm, Selaginella moellendorffii; Bd, Brachypodium distachyon; Sb, Sorghum bicolor; Mt, Medicago truncatula; Vv, Vitis vinifera; $\mathrm{Cp}$, Carica papaya; At, Arabidopsis thaliana; Os, Oryza sativa; Pt, Populus trichocarpa; Abbreviations: $\mathrm{Nl}$, not identified.

Additional file 9: Chromosomal locations of rice and poplar APC subunits and activators. Chromosome numbers are indicated at the bottom of each chromosome. Paralogs are linked by dashed lines. A, chromosomal positions of genes in rice. B, chromosomal positions of genes in poplar. Seven poplar genes were assigned to scaffolds.

Additional file 10: Neighbor-joining tree inferred from Poissoncorrected evolutionary distances for genes involved in the cell cycle and multiple sequence alignments of plant TPR subunits proteins. The TPR subunits (A) and activator gene family (B). The abbreviations of species names are as follows: At, Arabidopsis thaliana; Pt, Populus trichocarpa; Os, Oryza sativa; Vv, Vitis vinifera; Sb, Sorghum bicolor; Pp, Physcomitrella patens; Sm, Selaginella moellendorffii; Msp, Micromonas sp; Osp, Ostreococcus sp; Csp, Chlorella sp; Vc, Volvox carteri; Cm,

Cyanidioschyzon merolae; Zm, Zea mays; So, Saccharum officinarum; Mt, Medicago truncatula.
Additional file 11: Phylogenetic relationships between Plants and algae.

Additional file 12: OsCDC23_2 genomic sequence. Exon (yellow boxes), intron and possible exon (gray boxes). Sequencing result frameshift discarded.

Additional file 13: Pattern of internal domain duplications in Arabidopsis TPR subunits. The intensity of shading reflects the alignment score, with a dark shading for higher scores. The numbers on each axis indicate the domains in $\mathrm{N}$-to- $\mathrm{C}$ terminal orientation within the repeat.

Additional file 14: Expression patterns of rice APC genes based on Genevestigator. Expression patterns of OsAPC2, OsAPC7, OsAPC10, OSAPC11_1 and OSAPC13 are shown in different tissues (A) and developmental stages (B).

Additional file 15: Summary statistics for Ka and Ks. http://services. cbu.uib.no/tools/kaks.

Additional file 16: Promoter analysis. The upstream 1000 bp regions of all rice APC subunits genes were considered to contain the full length promoters. Table 2, motifs found in the promoters and their occurrences. Table 3, motifs found in each duplicated gene (in red, same motifs).

\section{Acknowledgements}

We are grateful to Dr. Martha Sorenson for critical reading and language revision of the manuscript and Rodrigo T. Lourenço for providing the poplar RNA samples. M.F.L. is indebted to Conselho Nacional de Desenvolvimento Científico e Tecnológico (CNPq), Fundação de Amparo à Pesquisa do Rio de Janeiro (FAPERJ) and BELSPO (Belgian Science Policy) for graduate and posdoctoral fellowships. P.C.G.F and A.S.H receive support from CNPq research grants. We are also thankful to Natura for financial support.

\section{Author details}

'Laboratório de Biologia Molecular de Plantas, Instituto de Bioquímica Médica, CCS, Cidade Universitária - Ilha do Fundão, CEP 21941-590, Rio de Janeiro, RJ, Brasil. ${ }^{2}$ Centro de Genômica e Fitomelhoramento, Departamento de Fitotecnia, Faculdade de Agronomia Eliseu Maciel, Universidade Federal de Pelotas, Campus Universitário s/n - Capão do Leão, CEP 90001-970, Pelotas, RS, Brasil. ${ }^{3}$ Stockholm Bioinformatics Center, Center for Biomembrane Research, Department of Biochemistry and Biophysics, Stockholm University, 106, 91,Stockholm, Sweden.

\section{Authors' contributions}

MFL carried out the molecular genetic studies, analyzed the data and drafted the manuscript. NBE, CP, RS, CR, TB, LV, AE and ACO carried out experiments and analyzed the data. ASH and PCGF conceived of the study, and participated in its design and coordination and helped to draft the manuscript. All authors read and approved the final manuscript.

Received: 21 September 2010 Accepted: 18 November 2010 Published: 18 November 2010

\section{References}

1. Joubes J, Chevalier C, Dudits D, Heberle-Bors E, Inze D, Umeda M, et al: CDK-related protein kinases in plants. Plant Mol Biol 2000, 43:607-620.

2. Furuno $\mathrm{N}$, den EN, Pines J: Human cyclin $A$ is required for mitosis until mid prophase. J Cell Biol 1999, 147:295-306.

3. Gong D, Pomerening JR, Myers JW, Gustavsson C, Jones JT, Hahn AT, et al: Cyclin $\mathrm{A} 2$ regulates nuclear-envelope breakdown and the nuclear accumulation of cyclin B1. Curr Biol 2007, 17:85-91.

4. den Elzen N, Pines J: Cyclin A is destroyed in prometaphase and can delay chromosome alignment and anaphase. J Cell Biol 2001, 153:121-136.

5. Irniger S, Piatti S, Michaelis C, Nasmyth K: Genes involved in sister chromatid separation are needed for B-type cyclin proteolysis in budding yeast. Cell 1995, 81:269-278. 
6. King RW, Peters JM, Tugendreich S, Rolfe M, Hieter P, Kirschner MW: A $20 \mathrm{~S}$ complex containing CDC27 and CDC16 catalyzes the mitosis-specific conjugation of ubiquitin to cyclin B. Cell 1995, 81:279-288.

7. Sudakin V, Ganoth D, Dahan A, Heller H, Hershko J, Luca FC, et al: The cyclosome, a large complex containing cyclin-selective ubiquitin ligase activity, targets cyclins for destruction at the end of mitosis. Mol Biol Cell 1995, 6:185-197.

8. Tugendreich S, Tomkiel J, Earnshaw W, Hieter P: CDC27Hs colocalizes with $\mathrm{CDC16Hs}$ to the centrosome and mitotic spindle and is essential for the metaphase to anaphase transition. Cell 1995, 81:261-268.

9. Yoon HJ, Feoktistova A, Wolfe BA, Jennings IL, Link AJ, Gould KL: Proteomics analysis identifies new components of the fission and budding yeast anaphase-promoting complexes. Curr Biol 2002, 12:2048-2054.

10. Zachariae W, Schwab M, Nasmyth K, Seufert W: Control of cyclin ubiquitination by CDK-regulated binding of Hct1 to the anaphase promoting complex. Science 1998, 282:1721-1724.

11. Kramer ER, Scheuringer N, Podtelejnikov AV, Mann M, Peters JM: Mitotic regulation of the APC activator proteins CDC2O and CDH1. Mol Biol Cell 2000, 11:1555-1569.

12. Pfleger $C M$, Kirschner MW: The KEN box: an APC recognition signal distinct from the D box targeted by Cdh1. Genes Dev 2000, 14:655-665.

13. Glotzer M, Murray AW, Kirschner MW: Cyclin is degraded by the ubiquitin pathway. Nature 1991, 349:132-138.

14. Littlepage $L E$, Ruderman JV: Identification of a new $A P C / C$ recognition domain, the $\mathrm{A}$ box, which is required for the Cdh1-dependent destruction of the kinase Aurora-A during mitotic exit. Genes Dev 2002, 16:2274-2285.

15. Reimann JD, Freed E, Hsu JY, Kramer ER, Peters JM, Jackson PK: Emi1 is a mitotic regulator that interacts with $\mathrm{Cdc} 20$ and inhibits the anaphase promoting complex. Cell 2001, 105:645-655.

16. Miller JJ, Summers MK, Hansen DV, Nachury MV, Lehman NL, Loktev A, et al: Emi1 stably binds and inhibits the anaphase-promoting complex/ cyclosome as a pseudosubstrate inhibitor. Genes Dev 2006, 20:2410-2420.

17. Baker DJ, Dawlaty MM, Galardy P, van Deursen JM: Mitotic regulation of the anaphase-promoting complex. Cell Mol Life Sci 2007, 64:589-600

18. Peters JM: The anaphase-promoting complex: proteolysis in mitosis and beyond. Mol Cell 2002, 9:931-943.

19. Jeganathan KB, Malureanu L, van Deursen JM: The Rae1-Nup98 complex prevents aneuploidy by inhibiting securin degradation. Nature 2005, 438:1036-1039.

20. Kim AH, Bonni A: Thinking within the $D$ box: initial identification of Cdh1-APC substrates in the nervous system. Mol Cell Neurosci 2007, 34:281-287.

21. Eloy NB, Coppens F, Beemster GT, Hemerly AS, Ferreira PC: The Arabidopsis anaphase promoting complex (APC): regulation through subunit availability in plant tissues. Cell Cycle 2006, 5:1957-1965.

22. Serralbo O, Perez-Perez JM, Heidstra R, Scheres B: Non-cell-autonomous rescue of anaphase-promoting complex function revealed by mosaic analysis of HOBBIT, an Arabidopsis CDC27 homolog. Proc Natl Acad Sci USA 2006, 103:13250-13255.

23. Perez-Perez JM, Serralbo O, Vanstraelen M, Gonzalez C, Criqui MC, Genschik P, et al: Specialization of CDC27 function in the Arabidopsis thaliana anaphase-promoting complex (APC/C). Plant J 2008, 53:78-89.

24. Marrocco K, Thomann A, Parmentier Y, Genschik P, Criqui MC: The APC/C E3 ligase remains active in most post-mitotic Arabidopsis cells and is required for proper vasculature development and organization. Development 2009, 136:1475-1485.

25. Cui L, Wall PK, Leebens-Mack JH, Lindsay BG, Soltis DE, Doyle JJ, et al: Widespread genome duplications throughout the history of flowering plants. Genome Res 2006, 16:738-749.

26. Capron A, Okresz L, Genschik P: First glance at the plant APC/C, a highly conserved ubiquitin-protein ligase. Trends Plant Sci 2003, 8:83-89.

27. Fulop K, Tarayre S, Kelemen Z, Horvath G, Kevei Z, Nikovics K, et al: Arabidopsis anaphase-promoting complexes: multiple activators and wide range of substrates might keep APC perpetually busy. Cell Cycle 2005, 4:1084-1092.

28. Schultz J, Copley RR, Doerks T, Ponting CP, Bork P: SMART: a web-based tool for the study of genetically mobile domains. Nucleic Acids Res 2000, 28:231-234.
29. Passmore LA, McCormack EA, Au SW, Paul A, Willison KR, Harper JW, et al: Doc1 mediates the activity of the anaphase-promoting complex by contributing to substrate recognition. EMBO J 2003, 22:786-796.

30. Page AM, Aneliunas V, Lamb JR, Hieter P: In vivo characterization of the nonessential budding yeast anaphase-promoting complex/cyclosome components Swm1p, Mnd2p and Apc9p. Genetics 2005, 170:1045-1062.

31. Blilou I, Frugier F, Folmer S, Serralbo O, Willemsen V, Wolkenfelt $H$, et al: The Arabidopsis HOBBIT gene encodes a CDC27 homolog that links the plant cell cycle to progression of cell differentiation. Genes Dev 2002, 16:2566-2575.

32. Rensing $S A$, Lang $D$, Zimmer AD, Terry A, Salamov A, Shapiro $H$, et al: The Physcomitrella genome reveals evolutionary insights into the conquest of land by plants. Science 2008, 319:64-69.

33. Goff SA, Ricke D, Lan TH, Presting G, Wang R, Dunn M, et al: A draft sequence of the rice genome (Oryza sativa L. ssp. japonica). Science 2002, 296:92-100.

34. Jaillon O, Aury JM, Noel B, Policriti A, Clepet C, Casagrande A, et al: The grapevine genome sequence suggests ancestral hexaploidization in major angiosperm phyla. Nature 2007, 449:463-467.

35. Masuda HP, Ramos GB, de Almeida-Engler J, Cabral LM, Coqueiro VM, Macrini CM, et al: Genome based identification and analysis of the prereplicative complex of Arabidopsis thaliana. FEBS Lett 2004, 574:192-202.

36. Wang G, Kong H, Sun Y, Zhang X, Zhang W, Altman N, et al: Genome-wide analysis of the cyclin family in Arabidopsis and comparative phylogenetic analysis of plant cyclin-like proteins. Plant Physiol 2004, 135:1084-1099.

37. Mori Y, Yamamoto T, Sakaquchi N, Ishibashi T, Furukawa T, Kadota Y, et al: Characterization of the origin recognition complex (ORC) from a higher plant, rice (Oryza sativa L.). Gene 2005, 353:23-30.

38. Shultz RW, Tatineni VM, Hanley-Bowdoin L, Thompson WF: Genome-wide analysis of the core DNA replication machinery in the higher plants Arabidopsis and rice. Plant Physiol 2007, 144:1697-1714.

39. Lammens T, Boudolf V, Kheibarshekan L, Zalmas LP, Gaamouche T, Maes S, et al: Atypical E2F activity restrains APC/CCCS52A2 function obligatory for endocycle onset. Proc Natl Acad Sci USA 2008, 105:14721-14726.

40. Blanc G, Hokamp K, Wolfe KH: A recent polyploidy superimposed on older large-scale duplications in the Arabidopsis genome. Genome Res 2003, 13:137-144.

41. Tuskan GA, Difazio S, Jansson S, Bohlmann J, Grigoriev I, Hellsten U, et al: The genome of black cottonwood, Populus trichocarpa (Torr. \& Gray). Science 2006, 313:1596-1604.

42. van $L R$, Clijsters $L$, Wolthuis $R$ : To cell cycle, swing the $A P C / C$. Biochim Biophys Acta 2008, 1786:49-59.

43. Peters JM: The anaphase promoting complex/cyclosome: a machine designed to destroy. Nat Rev Mol Cell Biol 2006, 7:644-656.

44. Jin L, Williamson A, Banerjee S, Philipp I, Rape M: Mechanism of ubiquitinchain formation by the human anaphase-promoting complex. Cell 2008, 133:653-665.

45. Herzog F, Primorac I, Dube P, Lenart P, Sander B, Mechtler K, et al: Structure of the anaphase-promoting complex/cyclosome interacting with a mitotic checkpoint complex. Science 2009, 323:1477-1481.

46. Blatch GL, Lassle M: The tetratricopeptide repeat: a structural motif mediating protein-protein interactions. Bioessays 1999, 21:932-939.

47. D'Andrea LD, Regan L: TPR proteins: the versatile helix. Trends Biochem Sci 2003, 28:655-662.

48. Patthy L: Modular assembly of genes and the evolution of new functions. Genetica 2003, 118:217-231.

49. Zhang J: Evolution by gene duplication: an update. Trends Ecol Evol 2003, 318:292-298.

50. Babushok DV, Ostertag EM, Kazazian HH Jr: Current topics in genome evolution: molecular mechanisms of new gene formation. Cell Mol Life Sci 2007, 64:542-554.

51. Lynch M, Conery JS: The evolutionary fate and consequences of duplicate genes. Science 2000, 290:1151-1155.

52. Moore AD, Bjorklund AK, Ekman D, Bornberg-Bauer E, Elofsson A: Arrangements in the modular evolution of proteins. Trends Biochem Sci 2008, 33:444-451.

53. Vogel C, Teichmann SA, Pereira-Leal J: The relationship between domain duplication and recombination. J Mol Biol 2005, 346:355-365. 
54. Wilson CG, Kajander T, Regan L: The crystal structure of Nlpl. A prokaryotic tetratricopeptide repeat protein with a globular fold. FEBS $J$ 2005, 272:166-179

55. Schmidt EE, Davies CJ: The origins of polypeptide domains. Bioessays 2007, 29:262-270.

56. Bjorklund AK, Ekman D, Elofsson A: Expansion of protein domain repeats. PLoS Comput Biol 2006, 2:e114

57. Rice P, Longden I, Bleasby A: EMBOSS: the European Molecular Biology Open Software Suite. Trends Genet 2000, 16:276-277.

58. Vinardell JM, Fedorova E, Cebolla A, Kevei Z, Horvath G, Kelemen Z, et al: Endoreduplication mediated by the anaphase-promoting complex activator CCS52A is required for symbiotic cell differentiation in Medicago truncatula nodules. Plant Cell 2003, 15:2093-2105.

59. Vanstraelen M, Baloban $M, D a I O$, Cultrone A, Lammens T, Boudolf $V$, et al: APC/C-CCS52A complexes control meristem maintenance in the Arabidopsis root. Proc Natl Acad Sci USA 2009, 106:11806-11811.

60. Desnos T, Orbovic V, Bellini C, Kronenberger J, Caboche M, Traas J, et al: Procuste 1 mutants identify two distinct genetic pathways controlling hypocotyl cell elongation, respectively in dark- and light-grown Arabidopsis seedlings. Development 1996, 122:683-693.

61. Galbraith DW, Harkins KR, Knapp S: Systemic Endopolyploidy in Arabidopsis thaliana. Plant Physiol 1991, 96:985-989.

62. Gendreau E, Traas J, Desnos T, Grandjean O, Caboche M, Hofte H: Cellular basis of hypocotyl growth in Arabidopsis thaliana. Plant Physiol 1997, 114:295-305.

63. Edgar BA, Orr-Weaver TL: Endoreplication cell cycles: more for less. Cell 2001, 105:297-306.

64. Diffley JF: Regulation of early events in chromosome replication. Curr Biol 2004, 14:R778-R786.

65. Imai KK, Ohashi Y, Tsuge T, Yoshizumi T, Matsui M, Oka A, et al: The A-type cyclin CYCA2;3 is a key regulator of ploidy levels in Arabidopsis endoreduplication. Plant Cell 2006, 18:382-396.

66. Grennan AK: Genevestigator: Facilitating web-based gene-expression analysis. Plant Physiol 2006, 141:1164-1166.

67. Kwiatkowska D: Flowering and apical meristem growth dynamics. J Exp Bot 2009, 59:187-201.

68. Sabelli PA, Larkins BA: The contribution of cell cycle regulation to endosperm development. Sex Plant Reprod 2009, 22:207-219.

69. Tarayre S, Vinardell JM, Cebolla A, Kondorosi A, Kondorosi E: Two classes of the CDh1-type activators of the anaphase-promoting complex in plants: novel functional domains and distinct regulation. Plant Cell 2004, 16:422-434.

70. Kondorosi E, Kondorosi A: Endoreduplication and activation of the anaphase-promoting complex during symbiotic cell development. FEBS Lett 2004, 567:152-157.

71. Tatusov RL, Koonin EV, Lipman DJ: A genomic perspective on protein families. Science 1997, 278:631-637.

72. Pal M, Nagy O, Menesi D, Udvardy A, Deak P: Structurally related TPR subunits contribute differently to the function of the anaphasepromoting complex in Drosophila melanogaster. J Cell Sci 2007, 120:3238-3248.

73. Burton JL, Solomon MJ: D box and KEN box motifs in budding yeast $\mathrm{Hsl1p}$ are required for APC-mediated degradation and direct binding to Cdc20p and Cdh1p. Genes Dev 2001, 15:2381-2395.

74. Castro A, Vigneron S, Bernis C, Labbe JC, Lorca T: Xkid is degraded in a Dbox, KEN-box, and A-box-independent pathway. Mol Cell Biol 2003, 23:4126-4138.

75. Moore RC, Purugganan MD: The evolutionary dynamics of plant duplicate genes. Curr Opin Plant Biol 2005, 8:122-128.

76. Craig A, Ewan R, Mesmar J, Gudipati V, Sadanandom A: E3 ubiquitin ligases and plant innate immunity. J Exp Bot 2009, 60:1123-1132.

77. Frescas D, Pagano M: Deregulated proteolysis by the F-box proteins SKP2 and beta-TrCP: tipping the scales of cancer. Nat Rev Cancer 2008, 8:438-449

78. Pesin JA, Orr-Weaver TL: Regulation of APC/C activators in mitosis and meiosis. Annu Rev Cell Dev Biol 2008, 24:475-499.

79. Gagne JM, Downes BP, Shiu SH, Durski AM, Vierstra RD: The F-box subunit of the SCF E3 complex is encoded by a diverse superfamily of genes in Arabidopsis. Proc Natl Acad Sci USA 2002, 99:11519-11524.

80. The Arabdidopsis Genome Initiative: Analysis of the genome sequence of the flowering plant Arabidopsis thaliana. Nature 2000, 408:796-815.
81. Karpenahalli MR, Lupas AN, Soding J: TPRpred: a tool for prediction of TPR-, PPR- and SEL1-like repeats from protein sequences. BMC Bioinformatics 2007, 8:2.

82. Tamura K, Dudley J, Nei M, Kumar S: MEGA4: Molecular Evolutionary Genetics Analysis (MEGA) software version 4.0. Mol Biol Evol 2007, 24:1596-1599.

83. Higo K, Ugawa $\mathrm{Y}$, Iwamoto $\mathrm{M}$, Korenaga T: Plant cis-acting regulatory DNA elements (PLACE) database. Nucleic Acids Research 1999, 27:297-300.

84. Nemhauser JL, Mockler TC, Chory J: Interdependency of Brassinosteroid and Auxin Signaling in Arabidopsis. PLoS Biology 2004, 2:1460-1471.

85. Siltberg J, Liberles DA: A simple covarion-based approach to analyse nucleotide substitution rates. J Evol Biol 2002, 15:588-594.

86. Longemann J, Schell J, Willmitzer L: Improved method for the isolation of RNA from plant tissues. Anal Biochem 1987, 163:16-20.

doi:10.1186/1471-2229-10-254

Cite this article as: Lima et al.: Genomic evolution and complexity of the Anaphase-promoting Complex (APC) in land plants. BMC Plant Biology 2010 10:254.

\section{Submit your next manuscript to BioMed Central and take full advantage of:}

- Convenient online submission

- Thorough peer review

- No space constraints or color figure charges

- Immediate publication on acceptance

- Inclusion in PubMed, CAS, Scopus and Google Scholar

- Research which is freely available for redistribution
Biomed Central 